\title{
Tailoring Intercalant Assemblies at the Graphene-Metal Interface
}

\author{
Johannes Halle*, Nicolas Néel, Jörg Kröger \\ Institut für Physik, Technische Universität Ilmenau, D-98693 Ilmenau, Germany \\ E-mail: johannes.halle@tu-ilmenau.de \\ This document is the unedited Author's version of a Submitted Work that was subsequently accepted for \\ publication in Langmuir, copyright (c) American Chemical Society and the Division of Chemical Education, Inc., \\ after peer review. To access the final edited and published work see \\ https://pubs.acs.org/articlesonrequest/AOR-k9bCzvtT3V6WRd3q4UUw
}

\begin{abstract}
The influence of graphene on the assembly of intercalated material is studied using low-temperature scanning tunneling microscopy. Intercalation of $\mathrm{Pt}$ under monolayer graphene on $\mathrm{Pt}(111)$ induces a substrate reconstruction that is qualitatively different from the lattice rearrangement induced by metal deposition on $\mathrm{Pt}(111)$ and, specifically, the homoepitaxy of Pt. Alkali metals $\mathrm{Cs}$ and $\mathrm{Li}$ are used as intercalants for monolayer and bilayer graphene on $\mathrm{Ru}(0001)$. Atomically resolved topographic data reveal that at elevated alkali metal coverage $(2 \times 2) \mathrm{Cs}$ and $(1 \times 1) \mathrm{Li}$ intercalant structures form with respect to the graphene lattice.
\end{abstract}

\section{Introduction}

Superstructures at surfaces are ubiquitous. ${ }^{112}$ They may occur as reconstructions of the clean surface, as an ordered assembly of adsorbates, as a moiré pattern due to the lattice mismatch between adsorbate and substrate lattice or as an electronic superlattice.

In the case of adsorbates, superlattices form as the result of the balance between adsorbateadsorbate and adsorbate-substrate interactions. Exemplarily, when the adsorbate-adsorbate interaction is dominant characteristic molecular patterns may occur, ${ }^{3 / 5}$ while a stronger adsorbate-substrate coupling may be exploited for template effects, such as the guided adsorption on vicinal surfaces,, $6\left[14\right.$ on molecular platforms, $\frac{15[16}{16}$ and on moiré lattices. $\frac{17+39}{39}$ Adsorbate-substrate interactions mediated by surface state electrons ${ }^{40} \underline{45}$ and electronic moiré patterns ${ }^{46}$ were demonstrated to steer the adsorbate assembly as well.

The impetus to the work presented here was the addition of a third interaction and the exploration of a possible further tailoring of surface structures. To this end, graphene-covered metal surfaces, $\mathrm{Pt}(111)$ and $\mathrm{Ru}(0001)$, and the intercalation of $\mathrm{Pt}$ and alkali metals (Cs, Li) 
were used. The intercalated material experiences the coupling to the substrate as well as to the graphene. Pt intercalation under graphene on $\mathrm{Pt}(111)$ leads to a reconstruction of the $\mathrm{Pt}$ surface, which is related to the structures reported for homoepitaxial growth of $\mathrm{Pt}$ on pristine $\mathrm{Pt}(111)$. However, the presence of graphene induces a considerably extended longrange order of the reconstruction as well as a qualitative change in the underlying dislocation network. For Cs and Li intercalation on graphene-covered $\mathrm{Ru}(0001)$ alkali metal assemblies form with respect to the graphene lattice rather than to the metal substrate.

\section{Experimental Methods}

The experiments were performed in ultrahigh vacuum $\left(10^{-9} \mathrm{~Pa}\right)$ with a scanning tunneling microscope (STM) operated at $5 \mathrm{~K}$. Surfaces of $\mathrm{Pt}(111)$ and $\mathrm{Ru}(0001)$ were cleaned by repeated $\mathrm{Ar}^{+}$bombardment and annealing at $1200 \mathrm{~K}$ in $\mathrm{O}_{2}$ atmosphere $\left(4 \cdot 10^{-5} \mathrm{~Pa}\right)$. Graphene was prepared on the clean surfaces by thermal decomposition of $\mathrm{C}_{2} \mathrm{H}_{4}$. On $\mathrm{Ru}(0001)$ a second layer of graphene was formed by segregation of bulk C. ${ }^{47}$ Metal intercalation was performed by, first, depositing Pt from a hot filament, Cs and Li from commercial dispensers onto the respective metal surfaces at room temperature and, second, by annealing the sample at $1200 \mathrm{~K}(\mathrm{Pt}), 660 \mathrm{~K}(\mathrm{Cs}), 570 \mathrm{~K}(\mathrm{Li})$. Using this preparation protocol leads to the efficient intercalation of the deposited material, as previously shown for Cs, ${ }^{48} \mathrm{Li}$, $\frac{4950}{4}$ and $\mathrm{Ni} .50$ The annealing after deposition is particularly important for Cs and Pt since otherwise adsorbed clusters remain on graphene. $25|48| 51$ The intercalation is further evidenced by the concomitant weakening of the graphene moiré pattern as well as by STM images showing the atomically resolved graphene lattice. Cointercalation proceeded via the deposition of Li onto Cs-intercalated graphene on $\mathrm{Ru}(0001)$, followed by annealing. STM tips were fabricated from pure $\mathrm{Au}$ wire and trained in situ by annealing and field emission on a Au substrate. Topographic data were acquired with constant current and the bias voltage applied to the sample. STM images were processed using WSxM.

\section{Results and Discussion}

\section{$\operatorname{Pt}(111)$}

Annealing $\mathrm{Pt}(111)$ at temperatures exceeding $1330 \mathrm{~K}$ induces a hexagonal reconstruction of the surface. ${ }^{53154}$ STM studies showed that exposing Pt(111) to Pt vapor stabilizes a similar reconstruction already at $400 \mathrm{~K}$, which resembles a honeycomb network of protruding lines. $\frac{55}{5}$ These characteristic superstructures consist of domains where the bulk face-centered cubic (fcc) stacking is retained alternating with hexagonal close-packed (hcp) stacking regions. More recent calculations using the Frenkel-Kontorova model ${ }^{[56}$ showed that $\operatorname{Pt}(111)$ indeed teeters at the brink of a stability domain and may be triggered to reconstruct by small environmental changes. ${ }^{57}$ The $\mathrm{Pt}(111)$ reconstruction is related to the soliton reconstruction of $\mathrm{Au}(111)^{\sqrt[58]{61}}$ and likewise occurs upon deposition of $\mathrm{Co}, \underline{62} \mathrm{Cr}, \underline{63} \mathrm{Cu} \frac{64}{64} \operatorname{Pt}(111)$. 

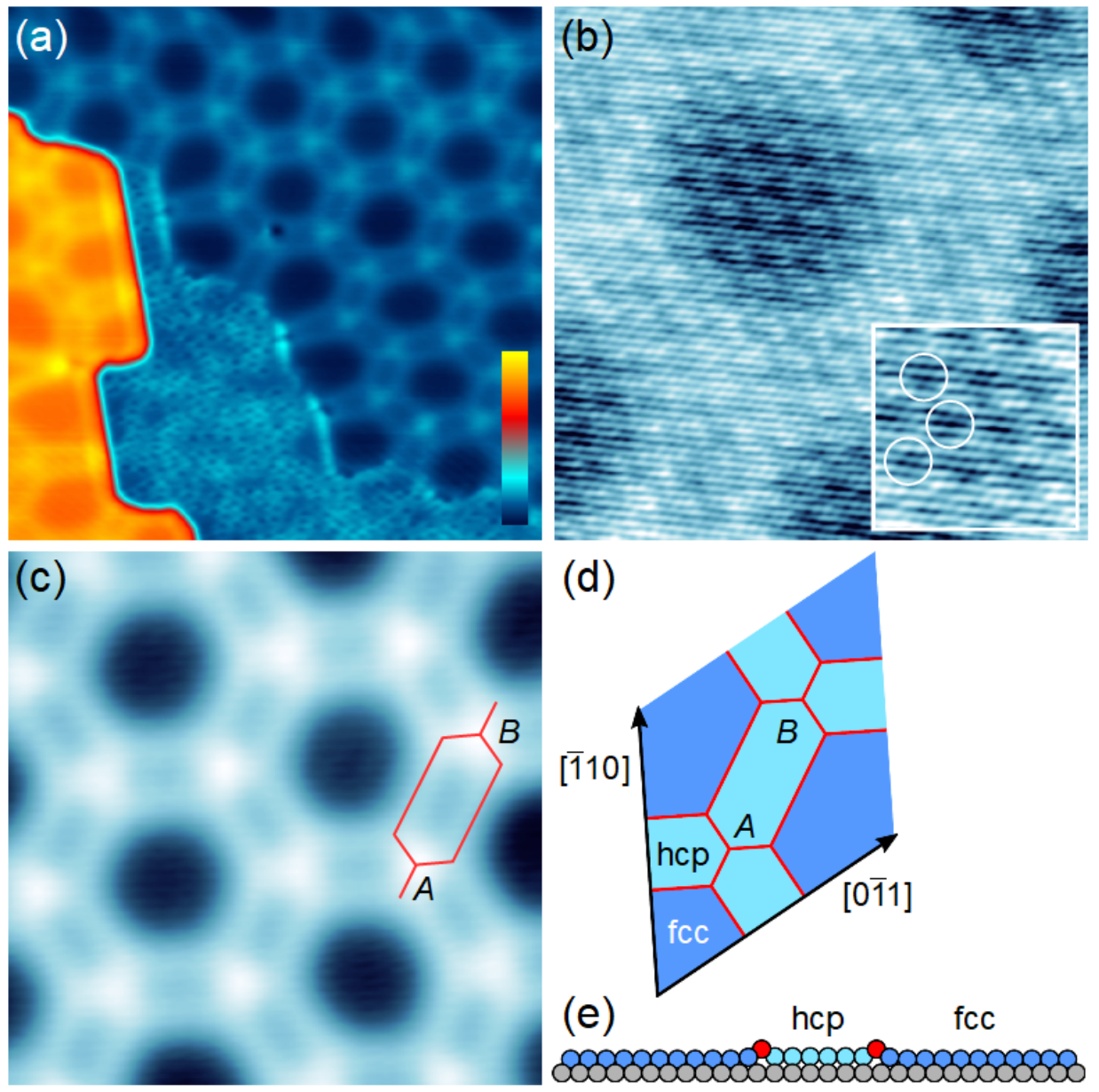

Figure 1: (Color online) Reconstruction of graphene-covered Pt(111) after Pt intercalation. (a) STM image of Pt-intercalated graphene on $\mathrm{Pt}(111)$ (bias voltage $V=1 \mathrm{~V}$, tunneling current $I=100 \mathrm{pA}$, size: $\left.40 \times 40 \mathrm{~nm}^{2}\right)$. Two adjacent $\operatorname{Pt}(111)$ terraces are visible. The honeycomb network is due to a reconstruction of the Pt(111) surface. (b) Atomically resolved close-up view of (a) showing the graphene lattice with parts of the reconstruction $\left(50 \mathrm{mV}, 100 \mathrm{pA}, 12 \times 12 \mathrm{~nm}^{2}\right)$. Inset: Close-up view of (b) with circles indicating a moiré pattern $\left(3.5 \times 3.5 \mathrm{~nm}^{2}\right)$. (c) STM image of the $\operatorname{Pt}(111)$ reconstruction network showing the characteristic fcc (hexagonal dark depressions), hcp (stripelike depressions enclosed by bright double lines) stacking domains $\left(1 \mathrm{~V}, 100 \mathrm{pA}, 19 \times 19 \mathrm{~nm}^{2}\right)$. The triangle-shaped bright protrusions are the connectors of the network (see text). (d) Illustration of structural elements of the reconstruction network. Regions with hcp stacking are separated from fcc domains by pairs of lines that are oriented along [112] , [12̄1], [211] directions. The double lines intersect at $A$ and $B$. (e) Side view of first and second $\operatorname{Pt}(111)$ atomic layer along $\langle\overline{1} 10\rangle$. 30 surface $\mathrm{Pt}$ atoms reside atop 29 second-layer Pt atoms giving rise to fcc and hcp stacking regions with protruding double lines. 
Here, possible modifications of this reconstruction due to the presence of graphene are investigated. Figure 17 shows a representative STM image of two adjacent terraces of graphenecovered Pt(111) after Pt intercalation. Most obvious is a regular honeycomb superstructure that extends over a few hundred nanometers. It is characterized by a spatial period of $7.99 \pm 0.08 \mathrm{~nm}$ with parallel ridges separated by $1.98 \pm 0.10 \mathrm{~nm}$. This superstructure is assigned to a reconstruction of the $\mathrm{Pt}(111)$ surface due to its resemblance to previously reported reconstructions on that surface. ${ }^{53155]}$ In particular, the ridges will be referred to as double lines following conventional phrasing. .55565 . Deviating from the rather irregular network of double lines observed from the reconstruction induced by the homoepitaxial growth of Pt on clean $\operatorname{Pt}(111)^{[55[65}$ the presence of graphene apparently causes a regular and extended honeycomb reconstruction pattern. Before analyzing the superstructure in more detail two remarks are noteworthy. First, the STM image of Figure 1 a shows that regions without reconstruction may occur. A surface area on the lower terrace and close to a substrate edge does not exhibit a reconstructed surface. Second, the presence of monolayer graphene (MLG) can be inferred from the atomically resolved graphene lattice (Figure 1 b). As indicated by the circles in the inset to Figure 1 b, a moiré pattern with a spatial period of $1.00 \pm 0.02 \mathrm{~nm}$ is visible. The angle between the moiré and graphene lattice is $1.2^{\circ} \pm 1.1^{\circ}$, which is in accordance with the angle of $14.5^{\circ} \pm 0.7^{\circ}$ enclosed by graphene and the $\mathrm{Pt}(111)$ lattice. This graphene lattice orientation is observed on both reconstructed and unreconstructed surface regions since graphene spans these regions without discontinuity (Figure 1 1 ). A second orientation was observed on $\mathrm{Pt}(111)$ with a smaller spatial period of $0.79 \pm 0.02 \mathrm{~nm}$ and an angle of $-17.5^{\circ} \pm 0.8^{\circ}$ enclosed by the graphene and Pt lattices. These moiré patterns are characteristic for MLG on $\mathrm{Pt}(111) .66167$ The rather low number of graphene orientations observed here is probably due to the rather high annealing temperature used for the intercalation of the Pt atoms, which is in agreement with a previous report. $\underline{66}$

Figure 1c presents topographic data that unravel the structural elements of the observed reconstruction. The illustrations in Figures $1 \mathrm{~d}$,e help identify crystallographic directions and surface regions with different stackings. The double lines represent a pair of Shockley partial dislocations that separate hexagonal fcc stacking domains from smaller and elongated hcp-stacked regions. $\frac{55 \mid 65}{54}$ Thus, across a pair of Shockley partial dislocations one additional $\mathrm{Pt}$ atom is incorporated into the surface, which increases the surface atom density. In the one-dimensional illustration (Figure 1e) 30 surface Pt atoms reside on top of 29 second-layer Pt atoms along $\langle\overline{1} 10\rangle$ directions.

The arrangement of double lines in the honeycomb reconstruction leads to an isotropic compression of the $\mathrm{Pt}(111)$ surface. Previously, double lines were demonstrated to meet in two different manners leading to two different types of dislocation line connectors, the so-called bright and dark stars. $\frac{55 \mid 65}{5 r i g h t}$ stars are protruding intersections of three pairs of double lines, where central $\mathrm{Pt}$ atoms reside at on-top sites of $\mathrm{Pt}(111)$. They provide an energy gain by the annihilation of three point dislocations, which outweighs the energy costs for the accompanying stacking faults. ${ }^{55 \mid 65}$ Dark stars represent connectors that enclose extended hcp-stacked regions. ${ }^{55 \mid 65}$ Bright and dark stars reflect the threefold symmetry of $\operatorname{Pt}(111)$, which is due to the inequivalence of hcp and fcc sites. Indeed, in all previous reports the energy difference between hcp and fcc sites established a topological law that 
forces the two types of connectors - bright and dark stars - to alternate at the corners of the

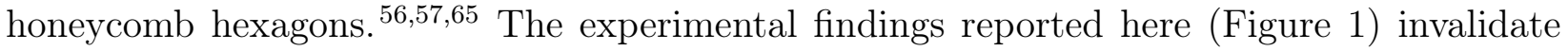
the topological law since the honeycomb reconstruction surprisingly exhibits protrusions at all connector sites of the dislocation network, labeled $A$ and $B$ in Figure 1c,d. A possible origin of this observation is discussed next.

The additional interaction of Pt surface atoms with graphene counteracts their coupling to the substrate, which facilitates the reconstruction. A similar argument was put forward to explain the stability of the high-temperature $\mathrm{Pt} / \mathrm{Pt}(111)$ reconstruction. In this case, a weaker bonding of the $\mathrm{Pt}$ surface layer was attributed to thermally excited vibrations. $.53|54| 57$ A second important consequence of the attraction between $\mathrm{Pt}$ and graphene is the reduction of stacking-dependent energy differences, especially between hcp and fcc sites. This aspect is corroborated by the remarkably large ratio of hcp-stacked to fcc-stacked areas, which is $\approx 0.78$ in the case presented here. In comparison, the purely temperature-induced reconstruction of $\mathrm{Pt}(111)$ at $1330 \mathrm{~K}$ yielded a hcp to fcc ratio of only 0.43 .53154 Additional deposition of $\mathrm{Pt}$, however, may further enhance this ratio. This was demonstrated in the homoepitaxial growth of $\mathrm{Pt}$ on $\mathrm{Pt}(111)$ at $400 \mathrm{~K}$ yielding a hcp to fcc ratio of $\approx 0.54 .55165$ Since the energy differences between the different stacking sites are reduced due to the presence of graphene, intralayer interactions become more important. Indeed, the strict alternation of bright and dark stars is overcome and the formation of bright stars as connectors of the observed reconstruction network is observed at all six double line intersections of a hexagon. This hints at a preference of bright stars over dark stars in the presence of graphene due to a lower formation energy. The above discussion unravels another important aspect that clarifies the role of graphene in the surface reconstruction. Graphene captures the intercalated Pt close to the substrate. The presence of $\mathrm{Pt}$ vapor was previously demonstrated to facilitate the reconstruction. $\underline{55[65}$

\section{$\operatorname{Ru}(0001)$}

The adsorption of alkali metals on surfaces has a longstanding tradition in surface science. 68 70 The promotion of catalytic reactions ${ }^{71 / 74}$ and the increase of electron emission rates ${ }^{75}$ belong to the appealing alkali-induced effects. It is therefore not surprising that many aspects of alkali metal adsorption have been studied, e. g., the geometric structure of superlattices, ${ }^{76}, 81$ vibrational quanta,,$\frac{82}{85}$ and lifetimes of electronic excitations ${ }^{86}$ On graphene, adsorption of alkali metals was shown to, e.g., tune the band gap opening between the graphene Dirac cones ${ }^{93[94}$ and modify the electronic transport. $\underline{.95196}$

More recently, the intercalation of alkali metals on graphene-covered surfaces has moved into a focus of surface science research. For instance, doping of graphene on $\operatorname{Ir}(111)$ may be controlled by the intercalation of Cs. ${ }^{97}$ In addition, the prediction of superconductivity in Li-decorated free-standing graphene ${ }^{98}$ has sparked investigations into electronic ${ }^{99 \mid 100}$ and structural $^{[49}$ as well as vibrational ${ }^{[50}$ properties.

In this section, structural aspects of $\mathrm{Cs}$, Li-interclated graphene on $\mathrm{Ru}(0001)$ are discussed. Figure 2 a shows an STM image of MLG and bilayer graphene (BLG) on Ru(0001). The pronounced superlattice is due to a moiré pattern that results from the strong MLG-

$\mathrm{Ru}(0001)$ hybridization. $101+103$ The moiré superlattice of MLG exhibits a spatial period of 

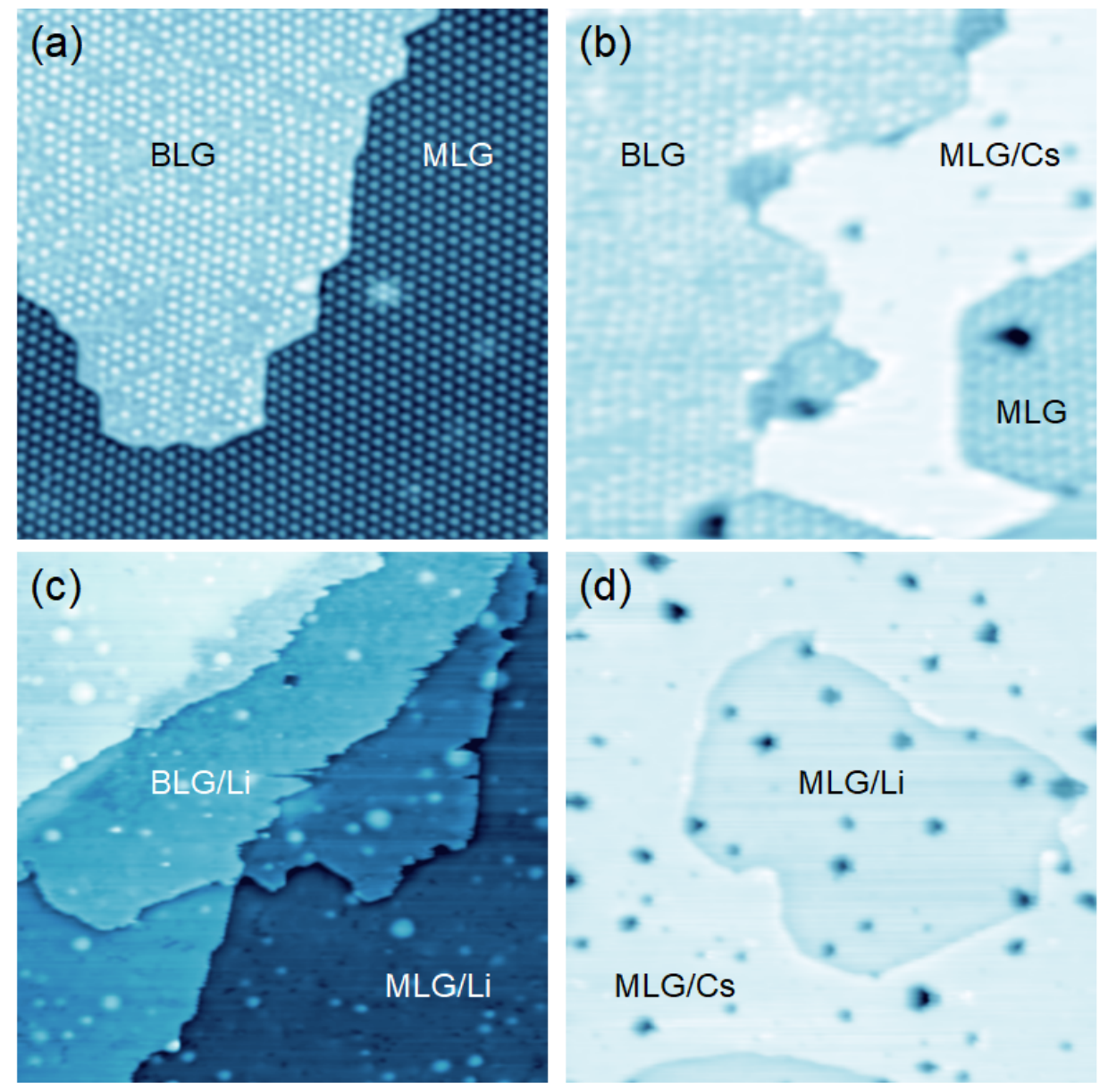

Figure 2: (Color online) Cs and Li intercalation phases on graphene-covered $\mathrm{Ru}(0001)$. (a) Monolayer (MLG) and bilayer (BLG) graphene on clean $\mathrm{Ru}(0001)(200 \mathrm{mV}, 100 \mathrm{pA}, 120 \times$ $120 \mathrm{~nm}^{2}$ ). The hexagonal superstructure is due to a moiré pattern. (b) Cs-intercalated graphene (MLG/Cs) between MLG and BLG domains $\left(200 \mathrm{mV}, 80 \mathrm{pA}, 90 \times 90 \mathrm{~nm}^{2}\right)$. (c) Liintercalated graphene $\left(300 \mathrm{mV}, 100 \mathrm{pA}, 300 \times 300 \mathrm{~nm}^{2}\right)$. Both MLG and BLG are intercalated and labeled as $\mathrm{MLG} / \mathrm{Li}$ and $\mathrm{BLG} / \mathrm{Li}$, respectively. The intercalated regions in (b) and (c) show an essentially vanishing moiré pattern. (d) Cointercalation of Cs and Li (200 mV, 90 pA, $\left.180 \times 180 \mathrm{~nm}^{2}\right)$. The separate intercalation phases are indicated. 
$3.02 \pm 0.05 \mathrm{~nm}$, in agreement with previous reports. 104109 The strong MLG buckling of $112 \pm 4$ pm mainly reflects the actual topography $101 / 109 \mid 110$ caused by the hybridization of graphene $\pi$-states with $\mathrm{Ru} d$-bands. $\frac{101 \mid 105}{10}$ The angle enclosed by the moiré and MLG lattice is $4.1^{\circ} \pm 1.0^{\circ}$, which corresponds to a calculated angle of $0.37^{\circ} \pm 0.09^{\circ}$ between the MLG and $\mathrm{Ru}(0001)$ lattice. The MLG spatial period and the moiré twist angles reflect an MLG lattice constant of $0.248 \mathrm{~nm}$, which corresponds to a $0.6 \%$ tensile stress in MLG. Similar conclusions have been drawn from earlier experimental work and density functional calculations. 101|104|107/109|111]

BLG flakes (Figure 2a) exhibit typical diameters of a few $100 \mathrm{~nm}$ and are observed to span several substrate terraces. Often, they occur at $\mathrm{Ru}(0001)$ step edges. BLG domains display a moiré pattern with a spatial period of $2.95 \pm 0.06 \mathrm{~nm}$ and an angle of $1.0^{\circ} \pm 0.6^{\circ}$ enclosed with the BLG lattice. The buckling of BLG is $119 \pm 1 \mathrm{pm}$, which is similar to the corrugation of MLG and in accordance with previous work. 105/108/110|111 In BLG regions, the analysis of the moiré pattern yields a lattice constant of $0.248 \mathrm{~nm}$ and a rotation angle of $0.33^{\circ} \pm 0.08^{\circ}$ with respect to $\mathrm{Ru}(0001)$ for the lower graphene layer. Within the uncertainty margins the values are comparable with those obtained for MLG and confirm that the moiré pattern of BLG on $\mathrm{Ru}(0001)$ is most likely due to the graphene- $\mathrm{Ru}(0001)$ interface. $107 / 110 / 112$ A second moiré pattern was previously reported for BLG on $\mathrm{Ru}(0001)$ when elevated $\mathrm{C}_{2} \mathrm{H}_{4}$ partial pressures were used for the graphene growth. ${ }^{108 / 109}$ In the present experiments the $\mathrm{C}_{2} \mathrm{H}_{4}$ partial pressure was two orders of magnitude lower than in the previous reports $\frac{108 / 109}{10}$ and, therefore, most likely impeded the formation of the second moiré pattern.

Intercalation of Cs (Figure 2b) and Li (Figure 2 $\mathrm{c}$ ) starts in MLG regions, while BLG intercalation is only observed at saturation coverage (Figure 2 $\mathrm{c}$ ). In order to determine the corresponding intercalation structures, MLG on Ru(0001) cointercalated by Cs and Li (Figure $2 \mathrm{~d}$ ) is considered. The cointercalated sample exhibits domains of different apparent heights, where the characteristic buckling of MLG on $\mathrm{Ru}(0001)$ is strongly reduced. This observation evidences the successful intercalation of graphene with $\mathrm{Cs}$ and $\mathrm{Li}$, which efficiently decouple MLG from the $\mathrm{Ru}$ surface. Consequently, the plane domains are attributed to separate Cs and Li intercalation phases, MLG/Cs and MLG/Li. The phase separation is most likely due to the lower delamination energy of Li-intercalated MLG. It is energetically favorable to form compact $\mathrm{Li}$ islands with a decreased graphene- $\mathrm{Ru}$ distance compared to the formation of mixed Cs-Li phases where the graphene- $\mathrm{Ru}$ separation would be increased due to the larger size of Cs.

The alkali metal intercalation structures may be deduced from atomically resolved STM images. Figure 3 a presents the transition between MLG/Cs and MLG/Li regions with a difference in the apparent height of $\approx 0.13 \mathrm{~nm}$ (inset to Figure $3 \mathrm{a}$ ). The intercalant assemblies are best seen from the atomically resolved close-up views of Figure 3 a. In Figure $3 \mathrm{~b}$ the honeycomb lattice of MLG is visible together with a regular hexagonal array of depressions. As corroborated by the Fourier transform (inset to Figure $3 \mathrm{~b}$ ) the array reflects a $(2 \times 2)$ lattice with respect to MLG. Alkali metals were previously shown to preferrably adopt the central part of a C hexagon of graphite or graphene, $\frac{113 \mid 114}{1}$ which is assumed here for MLG/Cs as well. This assumption is also reasonable with respect to the same conclusions drawn for a $(2 \times 2)$ Cs lattice observed from Cs-intercalated MLG on $\operatorname{Ir}(111) . \underline{48}$ Due to the lattice 

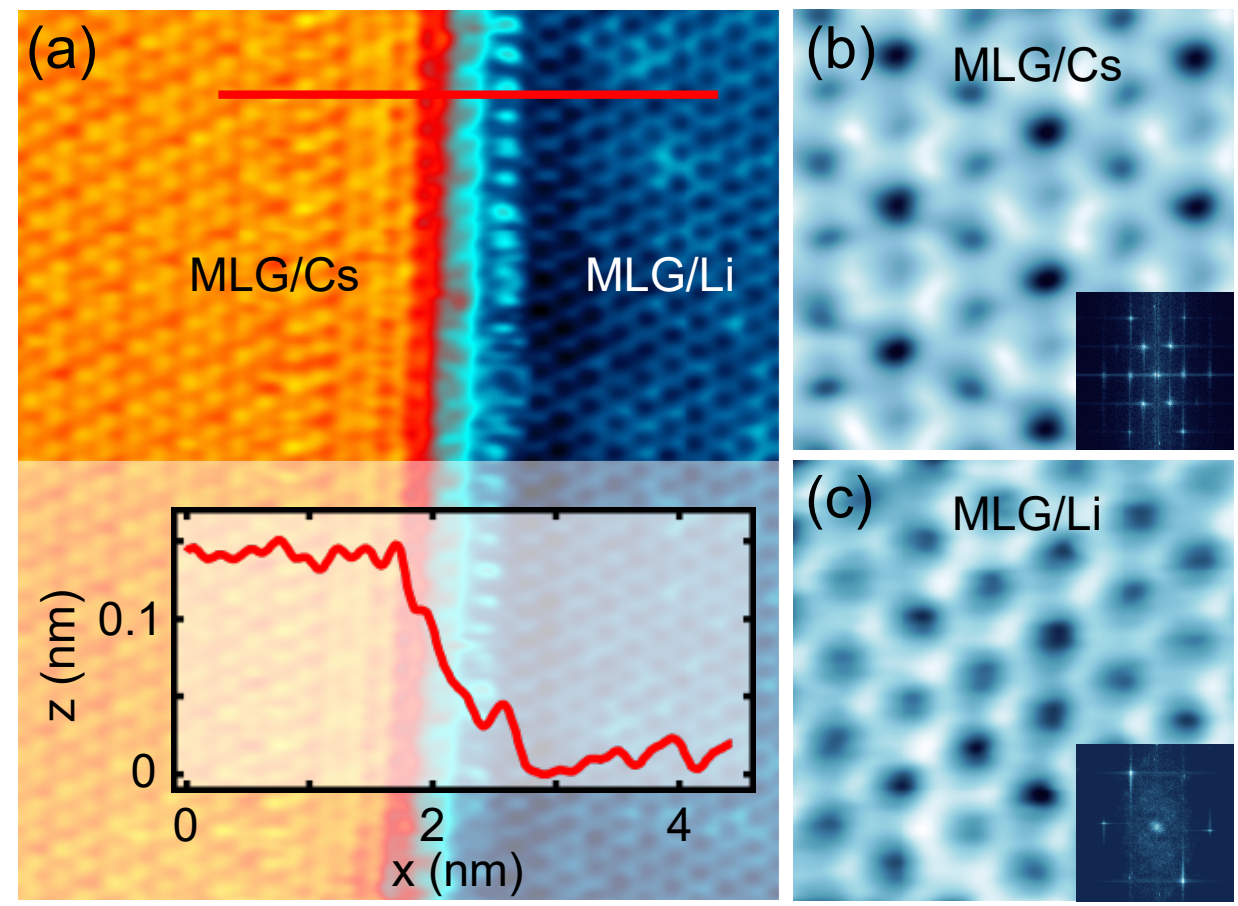

Figure 3: (Color online) Atomic structure of Cs and Li intercalation phases under MLG on $\mathrm{Ru}(0001)$. (a) STM image of adjacent Cs and Li intercalation phases $(200 \mathrm{mV}, 90 \mathrm{pA}$, $5.5 \times 8 \mathrm{~nm}^{2}$ ). Inset: Cross-sectional profile acquired along the line depicted in the top part of the STM image in (a). (b) Close-up view of Cs-intercalated domain with atomic resolution $\left(200 \mathrm{mV}, 90 \mathrm{pA}, 1.5 \times 1.5 \mathrm{~nm}^{2}\right)$. Inset: Fourier transform of $(\mathrm{b})\left(11.9 \times 11.9 \mathrm{~nm}^{-2}\right)$ with outer spots reflecting the $\mathrm{C}$ lattice of MLG. The inner spots indicate the $(2 \times 2)$ Cs ordered array. (c) Like (b) for a Li-intercalated region. Inset: Like the inset to (b) with identical spots for the MLG C lattice and the $(1 \times 1) \mathrm{Li}$ ordered structure. 
mismatch between MLG and $\mathrm{Ru}(0001)$, the intercalated Cs atoms do not always occupy $\mathrm{Ru}$ on-top sites, which were demonstrated to be favored sites for the $(2 \times 2)$ Cs superstructure on clean $\mathrm{Ru}(0001) .77$

Intercalation of $\mathrm{Li}$ under MLG on $\mathrm{Ru}(0001)$ leads to STM images as presented in Figure 3. The MLG honeycomb lattice is visible only and a $(1 \times 1) \mathrm{Li}$ assembly with respect to the MLG lattice is inferred (inset to Figure 3c); that is, each $\mathrm{C}$ hexagon is occupied by a $\mathrm{Li}$ atom. An alternative explanation would be a disordered phase, which has recently been reported from room temperature photoemission experiments on Li-covered $\operatorname{Ir}(111)$ and Liintercalated MLG on $\operatorname{Ir}(111) \cdot \frac{[15]}{}$ However, at $5 \mathrm{~K}$, which is the temperature of the experiments reported here, the suggested ordered $(1 \times 1) \mathrm{Li}$ intercalation phase is more plausible and agrees with the Li assembly observed at $6 \mathrm{~K}$ for Li-intercalated MLG on $\operatorname{Ir}(111) . \stackrel{48}{\text { For adsorption }}$ of $\mathrm{Li}$ on clean $\mathrm{Ru}(0001)$ a commensurate $(\sqrt{3} \times \sqrt{3}) \mathrm{R} 30^{\circ}-\mathrm{Li}$ phase was discovered at low coverage with Li atoms preferrably residing at threefold hcp hollow sites of $\mathrm{Ru}(0001) .{ }^{79}$ At larger submonolayer coverage incommensurate superstructures occur where Li atoms adopt different adsorption sites. 116

The intercalated $(2 \times 2) \mathrm{Cs}$ and $(1 \times 1) \mathrm{Li}$ phases observed here are formed with respect to the MLG lattice. Therefore, the preferred adsorption sites on clean $\mathrm{Ru}(0001)$ are no longer energetically favored, which demonstrates the strong impact of graphene on the energy landscape for alkali metal adsorption.

\section{Conclusions}

Graphene can tune the atomic structure of intercalated phases. The observed equilibrium assembly reflects the balance between couplings of the intercalant with graphene, the substrate and other intercalants. Graphene on $\operatorname{Pt}(111)$ weakens the adsorption site specificity for intercalated Pt atoms and modifies the adsorption energy landscape. The resulting surface reconstruction exhibits qualitative changes in the underlying dislocation network as well as an increased regularity and extension compared to reconstructions of pristine $\mathrm{Pt}(111)$. The intercalation of $\mathrm{Cs}$ and $\mathrm{Li}$ on graphene-covered $\mathrm{Ru}(0001)$ unravels a stronger impact of graphene on the intercalant assembly. Alkali metal lattices form superstructures that are commensurate with the graphene lattice, irrespective of their substrate adsorption site. The findings of this work may spark the tailoring of surface structures by the presence of graphene.

\section{Acknowledgements}

Financial support by the Deutsche Forschungsgemeinschaft through Grants No. KR 2912/101 and KR 2912/12 - 1 is acknowledged.

The authors declare no competing financial interest. 


\section{References}

[1] Ibach, H. Physics of surfaces and interfaces; Springer, 2006.

[2] Lüth, H. Solid surfaces, interfaces and thin films; Springer, 2015.

[3] Barth, J.; Costantini, G.; Kern, K. Engineering atomic and molecular nanostructures at surfaces. Nature 2005, 437, 671-679.

[4] Barth, J. V. Molecular Architectonic on Metal Surfaces. Annu. Rev. Phys. Chem. 2007, $58,375-407$.

[5] Wang, Y.; Wu, K.; Kröger, J.; Berndt, R. Review Article: Structures of phthalocyanine molecules on surfaces studied by STM. AIP Adv. 2012, 2, 041402.

[6] Shen, J.; Skomski, R.; Klaua, M.; Jenniches, H.; Manoharan, S. S.; Kirschner, J. Magnetism in one dimension: $\mathrm{Fe}$ on $\mathrm{Cu}(111)$. Phys. Rev. B 1997, 56, 2340-2343.

[7] Gambardella, P.; Blanc, M.; Brune, H.; Kuhnke, K.; Kern, K. One-dimensional metal chains on Pt vicinal surfaces. Phys. Rev. B 2000, 61, 2254-2262.

[8] Lin, J.-L.; Petrovykh, D. Y.; Kirakosian, A.; Rauscher, H.; Himpsel, F. J.; Dowben, P. A. Self-assembled Fe nanowires using organometallic chemical vapor deposition and $\mathrm{CaF}_{2}$ masks on stepped $\mathrm{Si}(111)$. Appl. Phys. Lett. 2001, 78, 829-831.

[9] Repain, V.; Baudot, G.; Ellmer, H.; Rousset, S. Two-dimensional long-range-ordered growth of uniform cobalt nanostructures on a $\mathrm{Au}(111)$ vicinal template. EPL (Europhysics Letters) 2002, 58, $730-736$.

[10] Kuhnke, K.; Kern, K. Vicinal metal surfaces as nanotemplates for the growth of lowdimensional structures. J. Phys.: Condens. Matter 2003, 15, S3311.

[11] Néel, N.; Kröger, J.; Berndt, R. Highly Periodic Fullerene Nanomesh. Adv. Mater. 2006, 18, 174-177.

[12] Néel, N.; Kröger, J.; Berndt, R. Fullerene nanowires on a vicinal gold surface. Appl. Phys. Lett. 2006, 88, 163101.

[13] Kröger, J.; Jensen, H.; Néel, N.; Berndt, R. Self-organization of cobalt-phthalocyanine on a vicinal gold surface revealed by scanning tunnelling microscopy. Surf. Sci. 2007, $601,4180-4184$.

[14] Chung, H. V.; Klevenz, M.; Lovrincic, R.; Neubrech, F.; Skibbe, O.; Pucci, A.; Nita, P.; Jalochowski, M.; Nagao, T. Studies on gold atom chains and lead nanowires on silicon vicinal surfaces. J. Phys. Conf. Ser. 2009, 187, 012025. 
[15] Barth, J. V.; Weckesser, J.; Cai, C.; Günter, P.; Bürgi, L.; Jeandupeux, O.; Kern, K. Building Supramolecular Nanostructures at Surfaces by Hydrogen Bonding. Angew. Chem. Int. Ed. 2000, 39, 1230-1234.

[16] Theobald, J. A.; Oxtoby, N. S.; Phillips, M. A.; Champness, N. R.; Beton, P. H. Controlling molecular deposition and layer structure with supramolecular surface assemblies. Nature 2003, 424, 1029 - 1031.

[17] Corso, M.; Auwärter, W.; Muntwiler, M.; Tamai, A.; Greber, T.; Osterwalder, J. Boron Nitride Nanomesh. Science 2004, 303, 217-220.

[18] Dil, H.; Lobo-Checa, J.; Laskowski, R.; Blaha, P.; Berner, S.; Osterwalder, J.; Greber, T. Surface Trapping of Atoms and Molecules with Dipole Rings. Science 2008, 319, 1824-1826.

[19] Berner, S.; Corso, M.; Widmer, R.; Groening, O.; Laskowski, R.; Blaha, P.; Schwarz, K.; Goriachko, A.; Over, H.; Gsell, S.; Schreck, M.; Sachdev, H.; Greber, T.; Osterwalder, J. Boron Nitride Nanomesh: Functionality from a Corrugated Monolayer. Angew. Chem. Int. Ed. 2007, 46, 5115-5119.

[20] Goriachko, A.; He,; Knapp, M.; Over, H.; Corso, M.; Brugger, T.; Berner, S.; Osterwalder, J.; Greber, T. Self-Assembly of a Hexagonal Boron Nitride Nanomesh on $\mathrm{Ru}(0001)$. Langmuir 2007, 23, 2928-2931.

[21] Goriachko, A.; He, Y. B.; Over, H. Complex Growth of NanoAu on BN Nanomeshes Supported by $\mathrm{Ru}(0001)$. J. Phys. Chem. C 2008, 112, 8147-8152.

[22] Järvinen, P.; Hämäläinen, S. K.; Ijäs, M.; Harju, A.; Liljeroth, P. Self-Assembly and Orbital Imaging of Metal Phthalocyanines on a Graphene Model Surface. J. Phys. Chem. C 2014, 118, 13320-13325.

[23] Mao, J.; Zhang, H.; Jiang, Y.; Pan, Y.; Gao, M.; Xiao, W.; Gao, H.-J. Tunability of Supramolecular Kagome Lattices of Magnetic Phthalocyanines Using Graphene-Based Moiré Patterns as Templates. J. Am. Chem. Soc. 2009, 131, 14136-14137.

[24] N'Diaye, A. T.; Gerber, T.; Busse, C.; Mysliveček, J.; Coraux, J.; Michely, T. A versatile fabrication method for cluster superlattices. New J. Phys. 2009, 11, 103045.

[25] Pan, Y.; Gao, M.; Huang, L.; Liu, F.; Gao, H.-J. Directed self-assembly of monodispersed platinum nanoclusters on graphene Moiré template. Appl. Phys. Lett. 2009, 95, 093106.

[26] Donner, K.; Jakob, P. Structural properties and site specific interactions of Pt with the graphene/Ru(0001) moiré overlayer. The Journal of Chemical Physics 2009, 131, 164701 . 
[27] Cavallin, A.; Pozzo, M.; Africh, C.; Baraldi, A.; Vesselli, E.; Dri, C.; Comelli, G.; Larciprete, R.; Lacovig, P.; Lizzit, S.; Alfè, D. Local Electronic Structure and Density of Edge and Facet Atoms at Rh Nanoclusters Self-Assembled on a Graphene Template. ACS Nano 2012, 6, 3034-3043.

[28] Sicot, M.; Bouvron, S.; Zander, O.; Rüdiger, U.; Dedkov, Y. S.; Fonin, M. Nucleation and growth of nickel nanoclusters on graphene Moiré on $\operatorname{Rh}(111)$. Appl. Phys. Lett. 2010, 96, 093115 .

[29] Pollard, A. et al. Supramolecular Assemblies Formed on an Epitaxial Graphene Superstructure. Angew. Chem. Int. Ed. 2010, 49, 1794-1799.

[30] Sutter, E.; Albrecht, P.; Wang, B.; Bocquet, M.-L.; Wu, L.; Zhu, Y.; Sutter, P. Arrays of $\mathrm{Ru}$ nanoclusters with narrow size distribution templated by monolayer graphene on Ru. Surf. Sci. 2011, 605, $1676-1684$.

[31] Roos, M.; Künzel, D.; Uhl, B.; Huang, H.-H.; Brandao Alves, O.; Hoster, H. E.; Gross, A.; Behm, R. J. Hierarchical Interactions and Their Influence upon the Adsorption of Organic Molecules on a Graphene Film. J. Am. Chem. Soc. 2011, 133, 9208-9211.

[32] Lu, J.; Yeo, P. S. E.; Zheng, Y.; Yang, Z.; Bao, Q.; Gan, C. K.; Loh, K. P. Using the Graphene Moiré Pattern for the Trapping of C60 and Homoepitaxy of Graphene. ACS Nano 2012, 6, 944-950.

[33] Bazarnik, M.; Brede, J.; Decker, R.; Wiesendanger, R. Tailoring Molecular SelfAssembly of Magnetic Phthalocyanine Molecules on Fe- and Co-Intercalated Graphene. ACS Nano 2013, 7, 11341-11349.

[34] Schulz, F.; Drost, R.; Hämäläinen, S. K.; Liljeroth, P. Templated Self-Assembly and Local Doping of Molecules on Epitaxial Hexagonal Boron Nitride. ACS Nano 2013, 7, 11121-11128.

[35] Järvinen, P.; Hämäläinen, S. K.; Banerjee, K.; Häkkinen, P.; Ijäs, M.; Harju, A.; Liljeroth, P. Molecular Self-Assembly on Graphene on SiO2 and h-BN Substrates. Nano Lett. 2013, 13, 3199-3204.

[36] Banerjee, K.; Kumar, A.; Canova, F. F.; Kezilebieke, S.; Foster, A. S.; Liljeroth, P. Flexible Self-Assembled Molecular Templates on Graphene. J. Phys. Chem. C 2016, 120, 8772-8780.

[37] Baltic, R.; Pivetta, M.; Donati, F.; Wäckerlin, C.; Singha, A.; Dreiser, J.; Rusponi, S.; Brune, H. Superlattice of Single Atom Magnets on Graphene. Nano Lett. 2016, 16, 7610-7615. 
[38] Avvisati, G.; Lisi, S.; Gargiani, P.; Pia, A. D.; Luca, O. D.; Pacilé, D.; Cardoso, C.; Varsano, D.; Prezzi, D.; Ferretti, A.; ; Betti, M. G. FePc Adsorption on the Moiré Superstructure of Graphene Intercalated with a Cobalt Layer. J. Phys. Chem. C 2017, 121, 1639-1647.

[39] Pivetta, M.; Rusponi, S.; Brune, H. Direct capture and electrostatic repulsion in the self-assembly of rare-earth atom superlattices on graphene. Phys. Rev. B 2018, 98, 115417.

[40] Kamna, M. M.; Stranick, S. J.; Weiss, P. S. Imaging Substrate-Mediated Interactions. Science 1996, 274, 118-119.

[41] Wahlström, E.; Ekvall, I.; Olin, H.; Walldén, L. Long-range interaction between adatoms at the $\mathrm{Cu}(111)$ surface imaged by scanning tunnelling microscopy. Appl. Phys. A 1998, 66, 1107-1110.

[42] Repp, J.; Moresco, F.; Meyer, G.; Rieder, K.-H.; Hyldgaard, P.; Persson, M. Substrate Mediated Long-Range Oscillatory Interaction between Adatoms: $\mathrm{Cu} / \mathrm{Cu}(111)$. Phys. Rev. Lett. 2000, 85, 2981-2984.

[43] Knorr, N.; Brune, H.; Epple, M.; Hirstein, A.; Schneider, M. A.; Kern, K. Long-range adsorbate interactions mediated by a two-dimensional electron gas. Phys. Rev. B 2002, 65,115420 .

[44] Hyldgaard, P.; Persson, M. Long-ranged adsorbate-adsorbate interactions mediated by a surface-state band. J. Phys.: Condens. Matter 2000, 12, L13 - L19.

[45] Silly, F.; Pivetta, M.; Ternes, M.; Patthey, F.; Pelz, J. P.; Schneider, W.-D. Creation of an Atomic Superlattice by Immersing Metallic Adatoms in a Two-Dimensional Electron Sea. Phys. Rev. Lett. 2004, 92, 016101.

[46] Gopakumar, T.; Néel, N.; Kröger, J.; Berndt, R. Spatial modulation of d states in a nanoscale Co island. Chem. Phys. Lett. 2009, 484, 59-63.

[47] Sutter, P. W.; Flege, J.-I.; Sutter, E. A. Epitaxial graphene on ruthenium. Nat. Mater. 2008, 7, 406.

[48] Petrović, M. et al. The mechanism of caesium intercalation of graphene. Nat. Commun. 2013, 4, 2772.

[49] Halle, J.; Néel, N.; Kröger, J. Filling the Gap: Li-Intercalated Graphene on $\operatorname{Ir}(111)$. J. Phys. Chem. C 2016, 120, 5067-5073.

[50] Halle, J.; Néel, N.; Fonin, M.; Brandbyge, M.; Kröger, J. Understanding and Engineering Phonon-Mediated Tunneling into Graphene on Metal Surfaces. Nano Lett. 2018, $18,5697-5701$. 
[51] Franz, D.; Blanc, N.; Coraux, J.; Renaud, G.; Runte, S.; Gerber, T.; Busse, C.; Michely, T.; Feibelman, P. J.; Hejral, U.; Stierle, A. Atomic structure of Pt nanoclusters supported by graphene/ $\operatorname{Ir}(111)$ and reversible transformation under $\mathrm{CO}$ exposure. Phys. Rev. B 2016, 93, 045426.

[52] Horcas, I.; Fernández, R.; Gómez-Rodríguez, J. M.; Colchero, J.; Gómez-Herrero, J.; Baro, A. M. WSXM: A software for scanning probe microscopy and a tool for nanotechnology. Rev. Sci. Instrum. 2007, 78, 013705.

[53] Sandy, A. R.; Mochrie, S. G. J.; Zehner, D. M.; Grübel, G.; Huang, K. G.; Gibbs, D. Reconstruction of the Pt(111) surface. Phys. Rev. Lett. 1992, 68, 2192-2195.

[54] Grübel, G.; Huang, K. G.; Gibbs, D.; Zehner, D. M.; Sandy, A. R.; Mochrie, S. G. J. Reconstruction of the $\mathrm{Pt}(111)$ surface: X-ray-scattering measurements. Phys. Rev. B 1993, 48, 18119-18139.

[55] Bott, M.; Hohage, M.; Michely, T.; Comsa, G. Pt(111) reconstruction induced by enhanced Pt gas-phase chemical potential. Phys. Rev. Lett. 1993, 70, 1489-1492.

[56] Kontorova, T.; Frenkel, J. On the theory of plastic deformation and twinning. II. Zh. Eksp. Teor. Fiz. 1938, 8, 1340-1348.

[57] Pushpa, R.; Narasimhan, S. Reconstruction of Pt(111) and domain patterns on closepacked metal surfaces. Phys. Rev. B 2003, 67, 205418.

[58] Harten, U.; Lahee, A. M.; Toennies, J. P.; Wöll, C. Observation of a Soliton Reconstruction of $\mathrm{Au}(111)$ by High-Resolution Helium-Atom Diffraction. Phys. Rev. Lett. 1985, 54, 2619-2622.

[59] Wöll, C.; Chiang, S.; Wilson, R. J.; Lippel, P. H. Determination of atom positions at stacking-fault dislocations on $\mathrm{Au}(111)$ by scanning tunneling microscopy. Phys. Rev. B 1989, 39, 7988-7991.

[60] Barth, J. V.; Brune, H.; Ertl, G.; Behm, R. J. Scanning tunneling microscopy observations on the reconstructed $\mathrm{Au}(111)$ surface: Atomic structure, long-range superstructure, rotational domains, and surface defects. Phys. Rev. B 1990, 42, 9307-9318.

[61] Narasimhan, S.; Vanderbilt, D. Elastic stress domains and the herringbone reconstruction on $\mathrm{Au}(111)$. Phys. Rev. Lett. 1992, 69, 1564-1567.

[62] Grütter, P.; Dürig, U. Quasidendritic growth of Co induced by localized reconstruction of Pt(111). Surf. Sci. 1995, 337, $147-152$.

[63] Zhang, L.; van Ek, J.; Diebold, U. Highly ordered nanoscale surface alloy formed through Cr-induced Pt(111) reconstruction. Phys. Rev. B 1998, 57, R4285-R4288.

[64] Holst, B.; Nohlen, M.; Wandelt, K.; Allison, W. Observation of an adlayer-driven substrate reconstruction in Cu-Pt(111). Phys. Rev. B 1998, 58, R10195-R10198. 
[65] Hohage, M.; Michely, T.; Comsa, G. Pt(111) network reconstruction: structure, growth and decay. Surf. Sci. 1995, 337, $249-267$.

[66] Merino, P.; Švec, M.; Pinardi, A. L.; Otero, G.; Martín-Gago, J. A. Strain-Driven Moiré Superstructures of Epitaxial Graphene on Transition Metal Surfaces. ACS Nano 2011, 5, 5627-5634.

[67] Gao, M.; Pan, Y.; Huang, L.; Hu, H.; Zhang, L. Z.; Guo, H. M.; Du, S. X.; Gao, H.-J. Epitaxial growth and structural property of graphene on $\operatorname{Pt}(111)$. Appl. Phys. Lett. 2011, 98, 033101.

[68] Bonzel, H. P., Bradshaw, A. M., Ertl, G., Eds. Physics and chemistry of alkali metal adsorption; Elsevier, 1989.

[69] Diehl, R. D.; McGrath, R. Structural studies of alkali metal adsorption and coadsorption on metal surfaces. Surf. Sci. Rep. 1996, 23, $43-171$.

[70] Diehl, R. D.; McGrath, R. Current progress in understanding alkali metal adsorption on metal surfaces. J. Phys.: Condens. Matter 1997, 9, 951 - 968.

[71] Ortega, J. E.; Oellig, E. M.; Ferrón, J.; Miranda, R. Cs and O adsorption on Si(100) $2 \times 1$ : A model system for promoted oxidation of semiconductors. Phys. Rev. B 1987, $36,6213-6216$.

[72] Tikhov, M.; Rangelov, G.; Surnev, L. Interaction of oxygen with Na-covered $\operatorname{Si}(100)$. Surf. Sci. 1990, 231, $280-288$.

[73] Faraci, G.; Pennisi, A. Ge(100) $2 \times 1$ substoichiometric oxidation states promoted by a Cs overlayer. Surf. Sci. 1998, 409, $46-56$.

[74] Davydov, S. Y. On the interaction of oxygen with a Cs-monolayer-covered $\operatorname{Si}(100)$ surface. Appl. Surf. Sci. 1999, 140, 58 - 62.

[75] Taylor, J. B.; Langmuir, I. The Evaporation of Atoms, Ions and Electrons from Caesium Films on Tungsten. Phys. Rev. 1933, 44, 423-458.

[76] Lindgren, S. A.; Walldén, L.; Rundgren, J.; Westrin, P.; Neve, J. Structure of $\mathrm{Cu}(111) p(2 \times 2)$ Cs determined by low-energy electron diffraction. Phys. Rev. B 1983, 28, 6707-6712.

[77] Over, H.; Bludau, H.; Skottke-Klein, M.; Ertl, G.; Moritz, W.; Campbell, C. T. Coverage dependence of adsorption-site geometry in the $\mathrm{Cs} / \mathrm{Ru}(0001)$ system: A low-energy electron-diffraction analysis. Phys. Rev. B 1992, 45, 8638-8649.

[78] Leatherman, G. S.; Diehl, R. D. Phase diagrams and rotated incommensurate phases of K, Rb, and Cs adsorbed on Ag(111). Phys. Rev. B 1996, 53, 4939-4946. 
[79] Gierer, M.; Over, H.; Bludau, H.; Ertl, G. Structural properties of ordered alkali metal overlayers: a LEED analysis of the $\mathrm{Ru}(0001)-(\sqrt{3} \times \sqrt{3}) \mathrm{R} 30^{\circ}$-Li phase in comparison with related systems. Surf. Sci. 1995, 33\%, $198-204$.

[80] von Hofe, T.; Kröger, J.; Berndt, R. Adsorption geometry of $\mathrm{Cu}(111)$-Cs studied by scanning tunneling microscopy. Phys. Rev. B 2006, 73, 245434.

[81] Ziegler, M.; Kröger, J.; Berndt, R.; Filinov, A.; Bonitz, M. Scanning tunneling microscopy and kinetic Monte Carlo investigation of cesium superlattices on $\mathrm{Ag}(111)$. Phys. Rev. B 2008, 78, 245427.

[82] He, P.; Jacobi, K. Vibrational analysis of cesium on $\mathrm{Ru}(0001)$. Phys. Rev. B 1996, 53, 3658-3661.

[83] Rusina, G. G.; Eremeev, S. V.; Echenique, P. M.; Benedek, G.; Borisova, S. D.; Chulkov, E. V. Vibrations of alkali metal overlayers on metal surfaces. J. Phys.: Condens. Matter 2008, 20, 224007.

[84] Kröger, J.; Bruchmann, D.; Lehwald, S.; Ibach, H. Adsorption of lithium on Mo(110): an EELS study of the adsorbate vibrations and substrate phonons. Surf. Sci. 2000, $449,227-235$.

[85] Kröger, J.; Lehwald, S.; Ibach, H. Surface dynamics of Mo(110)-H and Mo(110)-Li. Surf. Sci. 2003, 530, $170-174$.

[86] Bauer, M.; Pawlik, S.; Aeschlimann, M. Resonance lifetime and energy of an excited Cs state on $\mathrm{Cu}(111)$. Phys. Rev. B 1997, 55, 10040-10043.

[87] Ogawa, S.; Nagano, H.; Petek, H. Phase and Energy Relaxation in an Antibonding Surface State: Cs/Cu(111). Phys. Rev. Lett. 1999, 82, 1931-1934.

[88] Borisov, A. G.; Gauyacq, J. P.; Kazansky, A. K.; Chulkov, E. V.; Silkin, V. M.; Echenique, P. M. Long-Lived Excited States at Surfaces: $\mathrm{Cs} / \mathrm{Cu}(111)$ and $\mathrm{Cs} / \mathrm{Cu}(100)$ Systems. Phys. Rev. Lett. 2001, 86, 488-491.

[89] Gauyacq, J.; Borisov, A.; Kazansky, A. Impurity-induced localisation of the 2D surfacestate continuum on a metal surface. Appl. Phys. A 2004, 78, 141-147.

[90] Corriol, C.; Silkin, V. M.; Sánchez-Portal, D.; Arnau, A.; Chulkov, E. V.; Echenique, P. M.; von Hofe, T.; Kliewer, J.; Kröger, J.; Berndt, R. Role of Elastic Scattering in Electron Dynamics at Ordered Alkali Overlayers on $\mathrm{Cu}(111)$. Phys. Rev. Lett. 2005, 95, 176802.

[91] Kröger, J.; Becker, M.; Jensen, H.; von Hofe, T.; Néel, N.; Limot, L.; Berndt, R.; Crampin, S.; Pehlke, E.; Corriol, C.; Silkin, V.; Sánchez-Portal, D.; Arnau, A.; Chulkov, E.; Echenique, P. Dynamics of surface-localised electronic excitations studied with the scanning tunnelling microscope. Prog. Surf. Sci. 2007, 82, $293-312$. 
[92] Ziegler, M.; Kröger, J.; Berndt, R.; Borisov, A. G.; Gauyacq, J. P. Linewidth of a cesium adatom resonance on $\mathrm{Ag}(111)$. Phys. Rev. B 2009, 79, 075401.

[93] Papagno, M.; Rusponi, S.; Sheverdyaeva, P. M.; Vlaic, S.; Etzkorn, M.; Pacilé, D.; Moras, P.; Carbone, C.; Brune, H. Large Band Gap Opening between Graphene Dirac Cones Induced by Na Adsorption onto an Ir Superlattice. ACS Nano 2012, 6, 199-204.

[94] Matyba, P.; Carr, A.; Chen, C.; Miller, D. L.; Peng, G.; Mathias, S.; Mavrikakis, M.; Dessa, D. S.; Keller, M. W.; Kapteyn, H. C.; Murnane, M. Controlling the electronic structure of graphene using surface-adsorbate interactions. Phys. Rev. B 2015, 92, $041407(\mathrm{R})$.

[95] Chen, J.-H.; Jang, C.; Adam, S.; Fuhrer, M. S.; Williams, E. D.; Ishigami, M. Chargedimpurity scattering in graphene. Nat. Phys. 2008, 4, $377-381$.

[96] Woo, S.; Hemmatiyan, S.; Morrison, T. D.; Rathnayaka, K. D. D.; Lyuksyutov, I. F.; Naugle, D. G. Temperature-dependent transport properties of graphene decorated by alkali metal adatoms (Li, K). Appl. Phys. Lett. 2017, 111, 263502.

[97] Schumacher, S.; Wehling, T. O.; Lazić, P.; Runte, S.; Förster, D. F.; Busse, C.; Petrović, M.; Kralj, M.; Blügel, S.; Atodiresei, N.; Caciuc, V.; Michely, T. The Backside of Graphene: Manipulating Adsorption by Intercalation. Nano Lett. 2013, 13, 5013-5019.

[98] Profeta, G.; Calandra, M.; Mauri, F. Phonon-mediated superconductivity in graphene by lithium deposition. Nat. Phys. 2012, 8, $131-134$.

[99] McChesney, J. L.; Bostwick, A.; Ohta, T.; Seyller, T.; Horn, K.; González, J.; Rotenberg, E. Extended van Hove Singularity and Superconducting Instability in Doped Graphene. Phys. Rev. Lett. 2010, 104, 136803.

[100] Ludbrook, B. M. et al. Evidence for superconductivity in Li-decorated monolayer graphene. Proceedings of the National Academy of Sciences 2015, 112, 11795-11799.

[101] Wang, B.; Bocquet, M.-L.; Marchini, S.; Günther, S.; Wintterlin, J. Chemical origin of a graphene moire overlayer on $\mathrm{Ru}(0001)$. Phys. Chem. Chem. Phys. 2008, 10, 35303534 .

[102] Preobrajenski, A. B.; Ng, M. L.; Vinogradov, A. S.; Mårtensson, N. Controlling graphene corrugation on lattice-mismatched substrates. Phys. Rev. B 2008, 78, 073401.

[103] Jiang, D.-e.; Du, M.-H.; Dai, S. First principles study of the graphene $/ \mathrm{Ru}(0001)$ interface. J. Chem. Phys. 2009, 130, 074705.

[104] Marchini, S.; Günther, S.; Wintterlin, J. Scanning tunneling microscopy of graphene on Ru(0001). Phys. Rev. B 2007, 76, 075429.

[105] Sutter, E.; Acharya, D. P.; Sadowski, J. T.; Sutter, P. Scanning tunneling microscopy on epitaxial bilayer graphene on ruthenium (0001). Appl. Phys. Lett. 2009, 94, 133101. 
[106] Altenburg, S. J.; Kröger, J.; Wang, B.; Bocquet, M.-L.; Lorente, N.; Berndt, R. Graphene on $\mathrm{Ru}(0001)$ : Contact Formation and Chemical Reactivity on the Atomic Scale. Phys. Rev. Lett. 2010, 105, 236101.

[107] Que, Y.; Xiao, W.; Fei, X.; Chen, H.; Huang, L.; Du, S. X.; Gao, H.-J. Epitaxial growth of large-area bilayer graphene on Ru(0001). Appl. Phys. Lett. 2014, 104, 093110.

[108] Papagno, M. et al. Two Distinct Phases of Bilayer Graphene Films on Ru(0001). ACS Nano 2012, 6, 9299-9304.

[109] Dubout, Q.; Calleja, F.; Sclauzero, G.; Etzkorn, M.; Lehnert, A.; Claude, L.; Papagno, M.; Natterer, F. D.; Patthey, F.; Rusponi, S.; Pasquarello, A.; Brune, H. Giant apparent lattice distortions in STM images of corrugated $\mathrm{sp}^{2}$-hybridised monolayers. New J. Phys. 2016, 18, 103027.

[110] Wang, B.; Bocquet, M.-L. Interfacial coupling in rotational monolayer and bilayer graphene on $\mathrm{Ru}(0001)$ from first principles. Nanoscale 2012, 4, 4687-4693.

[111] Moritz, W.; Wang, B.; Bocquet, M.-L.; Brugger, T.; Greber, T.; Wintterlin, J.; Günther, S. Structure Determination of the Coincidence Phase of Graphene on $\mathrm{Ru}(0001)$. Phys. Rev. Lett. 2010, 104, 136102.

[112] Cui, Y.; Fu, Q.; Bao, X. Dynamic observation of layer-by-layer growth and removal of graphene on $\mathrm{Ru}(0001)$. Phys. Chem. Chem. Phys. 2010, 12, 5053-5057.

[113] DiVincenzo, D. P.; Mele, E. J. Cohesion and structure in stage-1 graphite intercalation compounds. Phys. Rev. B 1985, 32, 2538-2553.

[114] Uthaisar, C.; Barone, V. Edge Effects on the Characteristics of Li Diffusion in Graphene. Nano Lett. 2010, 10, 2838-2842.

[115] Pervan, P.; Lazić, P.; Petrović, M.; Šrut Rakić, I.; Pletikosić, I.; Kralj, M.; Milun, M.; Valla, T. Li adsorption versus graphene intercalation on $\operatorname{Ir}(111)$ : From quenching to restoration of the Ir surface state. Phys. Rev. B 2015, 92, 245415.

[116] Gierer, M.; Over, H.; Bludau, H.; Ertl, G. Incommensurate structures and epitaxial growth of $\mathrm{Li}$ on $\mathrm{Ru}(0001)$ : A quantitative low-energy electron-diffraction study. Phys. Rev. B 1995, 52, 2927-2934. 